\title{
Pulsation Properties of a Sample of Mira and OH/IR Stars
}

\author{
M. J. Gaylard and M. E. West \\ Hartebeesthoek Radio Astronomy Observatory, P O Box 443, \\ Krugersdorp 1740, South Africa
}

\begin{abstract}
Well-sampled light curves have been obtained for $1612-\mathrm{MHz}$ hydroxyl masers in Mira and OH/IR stars monitored with the Hartebeesthoek radio telescope. Pulsation modes are investigated via the ratios of the observed periods in the maser variations.
\end{abstract}

\section{Introduction}

The ranges of stellar masses and photospheric radii possible in Miras and OH/IR stars are such that pulsation could be occurring in the fundamental mode or the first or second overtones.

Stellar radii and masses are free parameters in theoretical pulsation models, but accurate distances are needed to derive the radii and masses of actual stars. The $1612 \mathrm{MHz}$ hydroxyl masers in OH/IR stars provide a method for determining individual stellar distances. The maser intensity follows the luminosity changes of the central star, but with a phase lag across the maser profile owing to the light travel time from the back to the front of the masing shell. Measurement of the phase lag gives the linear size of the shell. The ratio of the linear size to the angular size, determined interferometrically, then gives the distance to the star (van Langevelde et al. 1990).

A programme to determine distances to a set of Miras and OH/IRs using this method was initiated in 1985 . Fortnightly or weekly observations with the aim of measuring the phase lag across the $\mathrm{OH}$ shell are carried out with the 26-m Hartebeesthoek telescope. The MERLIN array is used to measure the angular sizes of the $\mathrm{OH}$ shells.

\section{Pulsation mode determination via period ratios}

The $\mathrm{OH}$ monitoring data can also be used in an alternative method for determining the pulsation modes which does not require the stellar distances to be found. The ratio of the observed periods $P$ of the primary and first overtone are compared to the ratios of the fundamental $P_{0}$ and first overtone $P_{1}$ and the first and second overtone $P_{2}$ predicted by models. The theoretical ratios form a set of curves as a function of period for each modelled mass (Ostlie \& Cox 1986).

The variations of the masers from eight $\mathrm{OH} / \mathrm{IR}$ stars in our sample were Fourier transformed to enable significant periodicities to be identified in the time series (Scargle 1982). No overtones were found in the power spectra of IK Tau 
$(P=470$ days $), \operatorname{IRC}-20197(P=640$ days $)$ and $0 H 358.2+0.5(P=1230$ days $)$. The periods and period ratios of the other stars are summarised in Table 1.

Table 1. Secondary periods $P_{\mathrm{S} n}$ with significance $\geq 2 \sigma$ present in the power spectra of the $\mathrm{OH}$ maser variations, and their ratios to the primary period $P_{\mathrm{P}}$.

\begin{tabular}{lrrrcc} 
star & $\begin{array}{r}P_{\mathrm{P}} \\
\text { days }\end{array}$ & $\begin{array}{r}P_{\mathrm{S} 1} \\
\text { days }\end{array}$ & $\begin{array}{r}P_{\mathrm{S} 2} \\
\text { days }\end{array}$ & $P_{\mathrm{P}} / P_{\mathrm{S} 1}$ & $P_{\mathrm{P}} / P_{\mathrm{S} 2}$ \\
\hline OH338.1+6.4 & 645 & 400 & - & 1.61 & - \\
WX Psc & 650 & 860 & 516 & 0.76 & 1.26 \\
OH1.3+1.0 & 945 & 545 & - & 1.73 & - \\
OH1.1-0.8 & 1310 & 720 & 540 & 1.82 & 2.43 \\
OH357.3-1.3 & 2000 & 1030 & - & 1.94 & -
\end{tabular}

For $\mathrm{OH} 338.1+6.4$ the sidelobe levels of the window function suggest that the apparent secondary period is a sampling artifact. WX Psc has the longest span of data and is the only object showing a significant period longer than the primary but distinguishable from long term trends.

The observed ratios $P_{\mathrm{P}} / P_{\mathrm{S} 1}$ are smaller than predicted by the Ostlie \& Cox (1986) models if $P_{\mathrm{P}}$ is identified with $P_{0}$. The predicted $P_{1} / P_{2}$ is of the order of 75 per cent of $P_{0} / P_{1}$, and is more consistent with the observed period ratios. Clearly this is dependent on the applicability of the models. There is a general trend for $P_{0} / P_{1}$ to increase with increasing period, in agreement with the model predictions. The period ratio for OH357.3-1.3 comes closest to the predicted $P_{0} / P_{1}$ ratio for fundamental mode pulsation. Pulsation in this mode would imply that the stellar mass is at least $5 \mathrm{M}_{\odot}$ and its radius is $\geq 1250 \mathrm{R}_{\odot}$. A switch from $P_{1}$ to $P_{0}$ as the star expands would be consistent with Wood's (1974) suggestion.

Fourier transforms of the $\mathrm{OH}$ maser luminosity variations show significant periods other than the primary in five out of eight cases. The period ratios are more consistent with the primary pulsation mode being the first overtone rather than the fundamental, with the possible exception of the 2000-day period OH357.3-1.3.

\section{References}

Ostlie, D. A., \& Cox, A. N. 1986, ApJ, 311, 864

Scargle, J. D. 1982, ApJ, 263, 835

van Langevelde, H. J., van der Heiden, R., \& van Schooneveld, C. 1990, A\&A, 239,193

Wood, P. R. 1974, ApJ, 190, 609 